\title{
Application Research of Tension Control System on Rotary Printing Press
}

\author{
Shengjiang Chen ${ }^{1}$, Chunfeng Zhang,"* \\ ${ }^{1}$ School of Nuclear Technology and Chemistry \& Biology,Hubei University of Science and \\ Technology,Xianning, Hubei,China \\ ${ }^{2}$ School of Foreign Languages,Hubei University of Science and Technology,Xianning, Hubei,China \\ *Corresponding author
}

Keywords: Rotary, Printing machine, Tension control system, Application

\begin{abstract}
A very important factor affecting the quality of printed matter is wheel printing machine tension control. Wheel printer control capability directly affects the paper tape tension facilities whether can run smoothly, and can adapt to different types of paper, in addition, the machine speed increase and decrease, paper roll diameter changes and automatic welding paper can remain stable, clear dot overprint without big changes. Based on dynamic principle, this paper analyzes the tension control in the printing machinery, and introduced the frequency conversion winding tension control system and application example on the wheel press. The reasonable system of high precision, simple control, adopt the system after the enterprise has obtained the very good economic benefits, is worth promoting.
\end{abstract}

\section{Introduction}

Rotation in printing is one of the most common graphic printing method, it not only fast printing speed, high plate cylinder seal resistance rate, especially suitable for mass printing, and print mode, rich layers, stereo sense is strong, can obtain shade have send, painted by information changes. Its product category is very broad: useful paper printing of books, newspaper, magazines and other publications, and money, securities, stamps, etc.; Useful thick cardboard printing paper, packaging; Useful plastic film printing of food, medicine and other flexible packaging bags and wall stickers, etc. As a result, it was published in graphic and packaging and printing has been occupying very important position in the business. In the coiling process of changes of the diameter of the drum is in operation, will cause the tension of coiled material change of the diameter of the change [1]. Tension is too small, coil is flabby wrinkle, will also go on lateral deflection. Tension is too big, can lead to coil stretched excessively, will appear in the longitudinal tension line, on the surface of the roller rod in uplift, may even make coil deformation and fracture. The main factors influencing the tension control has a mechanical loss, when the tensile elastic ratio, deceleration film roll tension change caused by inertia, coiling characteristics of the motor and drive device, etc. In the process of coiling, in order to ensure the production efficiency and improve the quality of the surface of the coil, constant tension is very necessary.

\section{The important significance and function of tension control}

Printing machine tension control is the core of the whole machine. Small, as long as stable tension control, tension of the printing press alignment precision and rejection rate is easy to control. Tension fluctuation and change had a great influence on the alignment precision; particularly equipment of the higher printing speed, tension control becomes more important. Therefore, if you want to make sure the printer print quality, efficiency and reliability, must be equipped with functional tension control system [1].

In the process of printing press tension fluctuate and change is more complex, if not timely and accurate under effective control, the comprehensive influence factors will form the tension of the 
machine parts in an irregular change model. Now its main influencing factors illustrate analysis is as follows:

The working characteristics of the printing press. Material in rolling process, and put the diameter of the coil winding and is constantly changing, the diameter of the changes will inevitably cause the change of strip tension [2]. Put the volume under the condition of the brake torque is constant, diameter decreases, and the tension will increase; and winding, on the other hand, if the winding at constant torque as the diameter increases, the tension will decrease. And material is heavier, the faster the speed, the change of strip tension. This is the intrinsic characteristics of the printer. Is one of the main factors of the change of the strip tension?

The precision of the printing press control. Press the manufacturing precision of the main components and assembly accuracy out-of-tolerance, is also the important factor of the tension caused by fluctuations. Such as base assembly flatness and straightness, wallboard and verticality of base and expressing ideas the levelness of the roller and parallelism between each other, and so on. Expressing ideas roller guide roller with many strict parallel with each other, not only in its rotation when they beat quantity deviation and deviation should also ensure quality dynamic and static balance within the scope of the specified value [2]. Otherwise their each roll a week, is expected to take tension will ensue tiny changes accordingly, finally comprehensive reflection on the machine, will lead to the tension produce irregular change. In addition, the printer's main drive system should be no gap, precision transmission, to ensure that each printing unit synchronization operation. If transmission synchronization error caused in printing process, also is bound to make changes in the tension of each unit. Therefore, it can be said that in the final analysis the precision of the machine itself standard is the basic guarantee for the tension implement effective control. Precision is high grade, high quality, stable tension control is easy; On the other hand, the precision level is too bad, bat out, even if equipped with the most advanced tension control system, may backfire, tension stability is difficult to obtain satisfactory results.

The stability of the printing press work. Printing machine in the process of non-stop automatically cutting process, material and cutting can make the machine already stable tension produce the interference of sudden change [3]. Machine running speed, the higher the disturbance. Equipment configuration of the tension control system should be able to quickly automatically according to the strip tension perturbation stochastic adjustment, restoring the tension in a timely manner to the original steady state.

The inhomogeneity of material. Strip the in homogeneity of inner material (such as fluctuations in elastic modulus, thickness along the length, the width direction, the eccentric material quality, etc. ) as well as the production environment temperature and humidity changes, will be a subtle influence on the whole machine tension fluctuation.

\section{The classification of the tension control system}

In recent years, China's domestic development rapidly, fully automatic computer color printing machine tension control of high precision, repeatable technology has been widely applied. While the printer is equipped with tension control system strengths and pattern diversity, but taken together, can largely be classified according to the following principles:

Open loop control system. The so-called open loop control is in the control system, there is no tension testing device and the feedback loop, or only test device without feedback loop control form. The way commonly used direct torque control mode, control the motor torque, the control need of mechanical loss, in the process of static moment of inertia, dynamic moment of inertia, deceleration compensation, poor control accuracy and stability [4], shown as Fig. 1. 


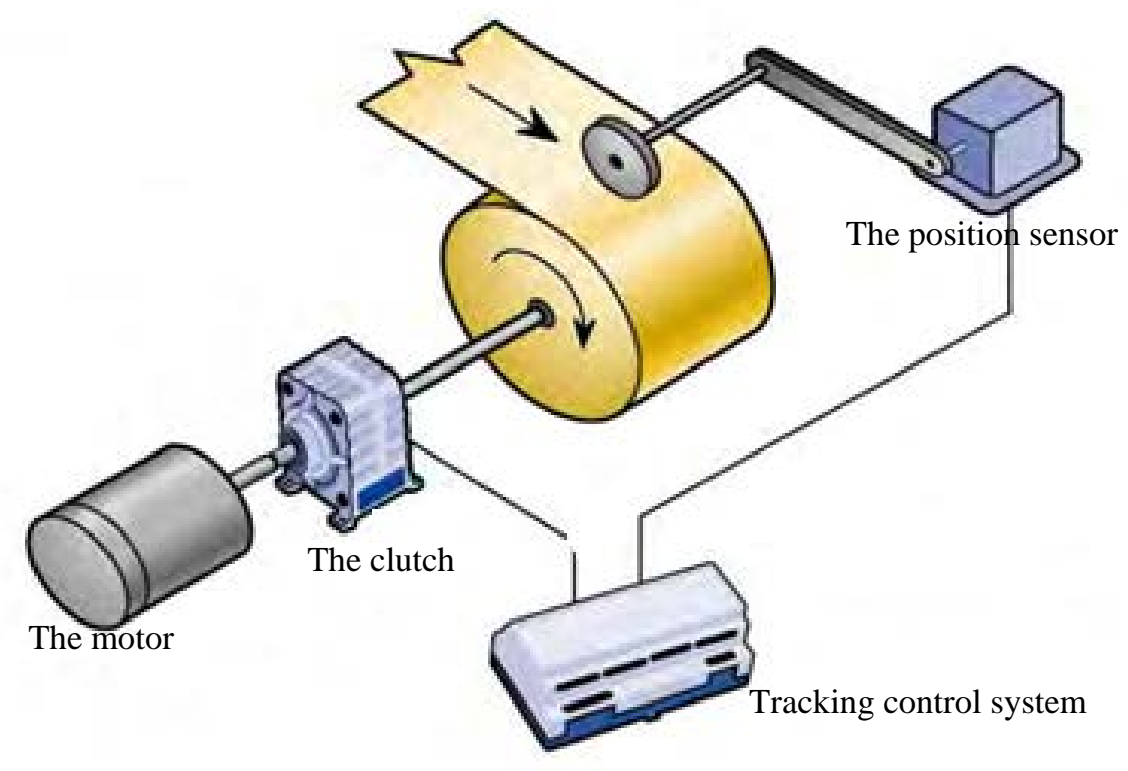

Fig. 1 Open loop control system

Closed loop control system. Closed-loop control is a detection device and the feedback loop control system. Closed-loop control of randomness is very strong, has the high control precision. A lot of feedback closed-loop control way, commonly used bridge type pressure sensor and the rotation axis of two kinds of tension sensor. Presented here with rotation shaft is the control scheme of the indirect tension test, which usually adopts the model of servo control, direct control of motor speed [4], shown as Fig. 2.

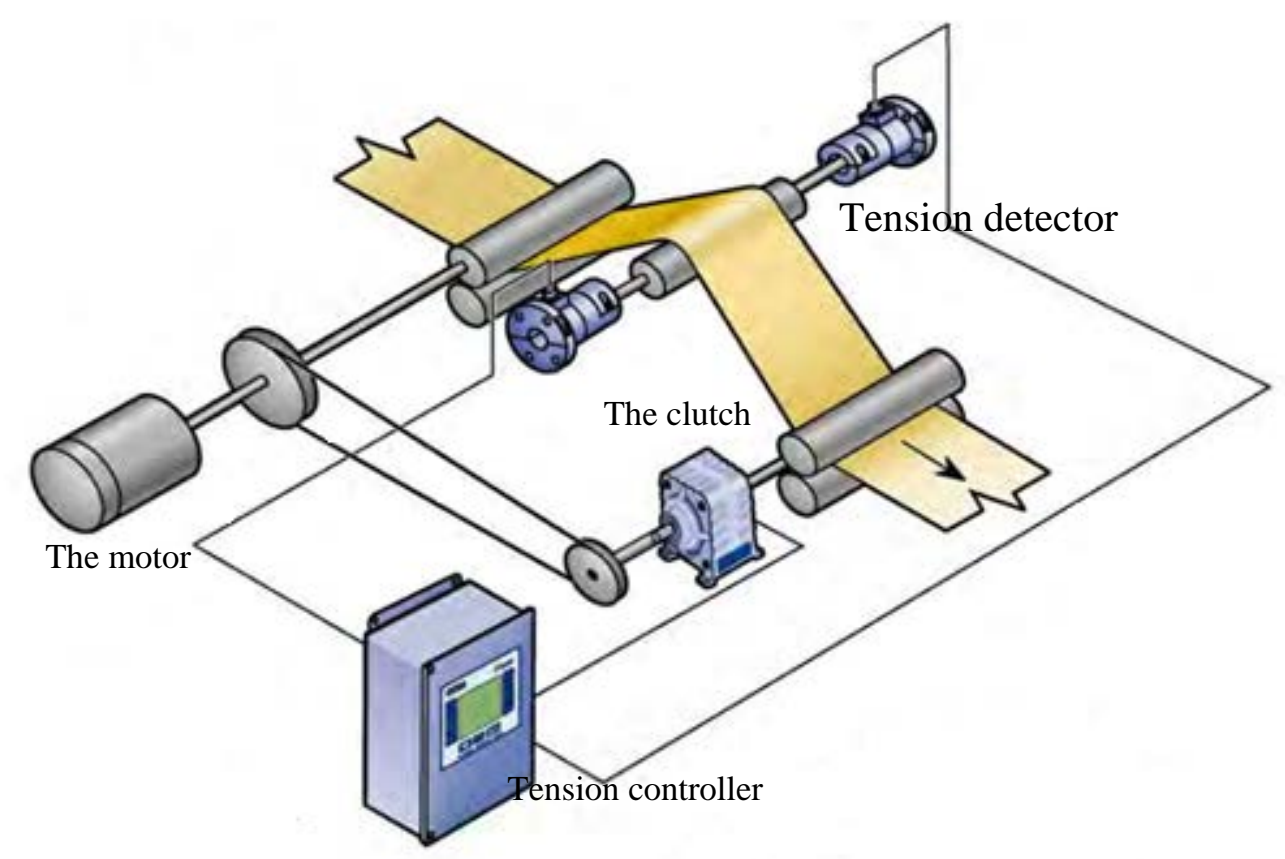

Fig. 2 Closed loop control system

\section{The application of rotation in control system of the printing press}

Wheel dynamic tension control system. When the cavity connected to the compressed air in cylinder, ACTS on the membrane tension for roller gravity vertical force and vertical force sum of the cylinder. Due to the rotational Angle is small, vertical component in the process of swing basically remain unchanged, therefore, directly change the cylinder pressure can adjust the film tension, 
tension has nothing to do with the roller diameter size [5]. In the winding process, when the tension change, wheel shaft corresponding swing, potentiometer with indirect tension change detection was adjusted by PID control winding speed, film tension constant.

In winding process is applied to the center, in addition to the above tension control, there are roller diameter increases, the roller under the condition of linear velocity is constant, the process of angular velocity decreases. At the beginning of the tensile strength of the coiled material effect on the rotation shaft and roll gravity, cylinder thrust phase equilibrium, rotation axis in the middle of the equilibrium position [6]. With the increase of roller diameter 6, wheel shaft 3 upward swing, turns the potentiometer 5 , the feedback signal deviates from the value of the original equilibrium voltage. Compared the signal with a given voltage signal, it is concluded that the deviation value, by the integral operation, adjust the motor speed, the dropping speed of the motor, the tension of coiled material to restore to the given value, wheel shaft back to its original equilibrium position. In the whole course of the winding with the increase of roller diameter, motor speed constant adjustment, membrane tension keep constant. Under normal circumstances, roller diameter range is $5 \sim 8$ times, commonly used servo drive mode, the speed range can reach about 10 times.

The rotation control mode. Running at high speed and low tension winding process, the requirement of tension is more outstanding, single floating roll control although can absorb some of the tension fluctuation, but in the process of the wheel shaft movement itself is caused by the inertia weight change can make the film tension change too [6]. Using double shaft relative to rotation, half as much as that of the single roller oscillating amplitude and because each wheel shaft can do much lighter weight, changes of tension caused by inertia small, too.

Rotation control system application. Wheel shaft in the tension of the automatic control process have the effect of testing tension changes, at the same time wheel shaft can absorb or buffer tension jump effect the stability of the system. Wheel shaft correct installation and use of the whole control system response sensitivity has a lot to do. Here is that need to be paid attention to when using the rotation system [7].

1) Wheel shaft under the working condition only one-way pressure, compressed gas cylinders can be unilateral, air flow can be through the atmosphere, so only need to control the pressure of the air inlet. Cylinder of air flow to install a throttle valve, can pass it to control the exhaust speed, make the cylinder has a back pressure, can prevent the cylinder before the rush too fast and impact. When using throttle valve should be adjusted to a suitable location, to two or more things tension detection sensitivity.

2) With low friction cylinder, cylinder can reduce the friction of piston and cylinder body, improve the cylinder response sensitivity. When the tensile strength of the effect on the rotation shaft has small fluctuations, also can produce fluctuations caused by the potentiometer to respond.

3) Relief valve must be used precision pressure regulating valve. Precision of high precision of the pressure regulating valve, can reduce the pressure of inlet gas caused by the fluctuation of the pendulum roller beating, lead to the change of the tension. At the same time, when the roller tension increased, counterclockwise torque than clockwise torque, the upward movement of cylinder, the cylinder inlet to the pressure regulating valve between the gas is compressed, pressure. Precision pressure regulating valve through the vent, the gas discharge rapidly, make the gas pressure inside the cylinder remains unchanged. In need taper control occasions, often transform precision pressure regulating valve to the E/P valve, air cylinder is controlled by different pressure to change the winding tension in the process of change.

4) Wheel shaft installed in the buffer device, can be at the end of the cylinder's stroke play the role of the buffer, to avoid the piston at the end of the trip the impact block, which can prolong the service life of cylinder.

5) Potentiometer with $360^{\circ}$ rotating conductive potentiometer, its effective pendulum Angle is $330^{\circ}$, rotation Angle should be less than $330^{\circ}$ and leave a little slack, lest and larger into dead zone affect the control gear 7 clearance should be small as far as possible at the same time, to ensure accuracy. 


\section{Summary}

Rotary printing press in the actual operation must be kept in the process of paper tape tension, the tension must maintain a constant can't big and small falling printing quality and even accidents. If the wheel printer paper tape tension is too large it is easy to cause the runner of the printing press overload which lead to broken paper accident; If the paper tape tension is too small, paper tape can't tighten, it is easy to cause paper, crinkle, more serious consequences can make paper to wheel on one side of the wall paper, or paper in the rubber roller or ink roller, causing the outage affected production waste. In this paper, the traditional wheel printer problems were analyzed, and put forward with the method of technical transformation to transform traditional wheel printing receive a unit for frequency conversion winding decoration, very good results have been achieved.

\section{Acknowledgements}

Subject Fund: the Knowledge Innovation Special Project of 2016 in Hubei Province (Natural Science Fund), Project Number: 2016CFC720.

\section{References}

[1] Q. Feng, Tension control technology of printing machine, the management and technology of small and medium-sized enterprise, 2009, vol. 7, pp. 15-18.

[2] X. Y. Deng, Tension control in paper machine drives control system of research, Microcomputer information, 2005, vol. 4, pp. 21-24. .

[3] G. L. Tang, Based on the wheel dynamic tension control and application, Packaging and food machinery, 2003, vol. 5, pp. 32-35.

[4] Y. Q. Sun, Web press tension control research, Packaging engineering, 2008, vol. 3, pp. 43-48.

[5] Z. T. Tao, Frequency control application research of electric drive automation on motor speed tension control system, Packaging and food machinery, 2010, vol. 1, pp. 54-58.

[6] Q. H. Zhao, A fuzzy adaptive of PID control in tension control, The application of packaging engineering, 2008, vol. 1, pp. 33-36.

[7] X. Y. Li, Tension control system analysis, programmable controller and factory automation, 2009, vol. 1, pp. 62-65. . 